\title{
Frontières
}

\section{Vie, mort et postérité sous Louis XIV à la lumière des oraisons funèbres du chancelier Michel Le Tellier}

\section{Luc-Normand Tellier}

Volume 14, numéro 2, printemps 2002

La mort prononcée

URI : https://id.erudit.org/iderudit/1073968ar

DOI : https://doi.org/10.7202/1073968ar

Aller au sommaire du numéro

Éditeur(s)

Université du Québec à Montréal

ISSN

1180-3479 (imprimé)

1916-0976 (numérique)

Découvrir la revue

Citer cet article

Tellier, L.-N. (2002). Vie, mort et postérité sous Louis XIV à la lumière des oraisons funèbres du chancelier Michel Le Tellier. Frontières, 14(2), 34-39. https://doi.org/10.7202/1073968ar
Résumé de l'article

Le chancelier Michel Le Tellier est à l'origine d'un des grands clans politiques de l'Ancien Régime en France. À sa mort, cinq oraisons funèbres ont été prononcées. Les deux plus célèbres, celles de Bossuet et de Fléchier, se distinguent profondément du fait qu'elles présentent des visions très différentes du sens qui doit être donné à la vie, à la mort et à la postérité. Étrangement, la position radicale adoptée par Bossuet en conformité avec la théologie catholique annonce la Révolution française, alors que la modération de Fléchier tente d'éviter de remettre en question l'idéal aristocratique du prolongement de l'individu dans sa descendance. 


\section{Résumé}

Le chancelier Michel Le Tellier est à l'origine d'un des grands clans politiques de l'Ancien Régime en France. À sa mort, cinq oraisons funèbres ont été prononcées. Les deux plus célèbres, celles de Bossuet et de Fléchier, se distinguent profondément du fait qu'elles présentent des visions très différentes du sens qui doit être donné à la vie, à la mort et à la postérité. Étrangement, la position radicale adoptée par Bossuet en conformité avec la théologie catholique annonce la Révolution française, alors que la modération de Fléchier tente d'éviter de remettre en question l'idéal aristocratique du prolongement de l'individu dans sa descendance.

Mots clés: oraisons funèbres - postérité mort-vie-Bossuet

\section{Abstract}

Chancellor Michel Le Tellier founded one of the most important political clans of the French Old Regime. When he died in 1685 , he was honored with five funeral orations. The two most famous ones were delivered by Bishops Bossuet and Fléchier who adopted quite opposite views on the sense life, death and posterity should be given with respect to each other. Strangely enough, the stand adopted by Bossuet on the basis of the Catholic creed heralded the French Revolution, while Fléchier's more moderate and conciliatory tone attempted not to challenge the aristocratic ideal of one's living on in his children and lineage.

Key words: funeral oration - posteritydeath-life-Bossuet

\section{Vie, mort et postérité sous Louis XIV à la lumière} des oraisons funèbres du chancelier Michel Le Tellier

\section{Luc-Normand Tellier, Ph.D., professeur, Département d'études urbaines et touristiques, UQÀM.}

Père de Louvois, allié habile de Mazarin sous la Fronde, créateur de l'armée monarchique sous Louis XIV, auteur du rétablissement, en 1679 , après quatre cent soixante ans, de l'enseignement du droit civil à l'Université de Paris et, à ce titre, père des facultés de droit de France et des pays de tradition française, le chancelier Michel Le Tellier est le fondateur du clan Le Tellier ${ }^{1}$ qui joua un rôle de premier plan en France pendant cent soixante ans, soit de la mort de Mazarin, en 1661, à la démission du poste de Premier ministre du duc de Richelieu$^{2}$, en 1821. Au lendemain de sa mort, survenue le 30 octobre 1685, Michel Le Tellier a eu l'honneur de faire l'objet de cinq oraisons funèbres ${ }^{3}$, alors que, par exemple, ni Colbert ni Louvois n'auront droit à un concert d'éloges aussi exceptionnel.

Deux des oraisons funèbres de Le Tellier, celles de Bossuet et de Fléchier, demeurent célèbres. Leur mise en parallèle permet de mieux saisir le sens que prenaient au Grand Siècle la vie, la mort et l'idée du prolongement de l'individu par-delà la mort à travers sa postérité.

\section{LA VIE}

Dans la théologie catholique qui anime la France de Louis XIV, la vie terrestre ne prend tout son sens que dans la perspective de la vie éternelle. Le but ultime de la vie d'ici-bas est de préparer à la vie éternelle et d'accéder au paradis. Du point de vue social, cela se réalise en se conformant aux rôles qui sont attribués à chacun tant à la naissance (le poids des rôles liés à la naissance est déterminant sous l'Ancien Régime) que, par la suite, au hasard des vocations et des cheminements individuels.

Parmi toutes les oraisons funèbres prononcées par Bossuet, celle de Le Tellier est la seule qui ne fasse aucun reproche, même voilé, au défunt. Selon Bossuet, Le Tellier est l'image même de la vertu et de la sagesse qui doivent animer tout personnage public, vertu et sagesse dont la mort même du chancelier témoigne. L'ouverture de son oraison le dit clairement:

Posside sapientiam, acquire prudentiam; arripe illam, et exaltabit te: glorificaberis ab ea, quum eam fueris amplexatus.

Possédez la vertu et acquérez la prudence: si vous la cherchez avec ardeur, elle vous élèvera et vous remplira de gloire, quand vous l'aurez embrassée. (Proverbes, 6, 7-8) 
Messeigneurs,

En louant l'homme incomparable dont cette illustre assemblée célèbre les funérailles et honore les vertus, je louerai la sagesse même; et la sagesse que je dois louer dans ce discours, n'est pas celle qui élève les hommes et qui agrandit les maisons, ni celle qui gouverne les empires, qui règle la paix et la guerre, et enfin qui dicte les lois, et qui dispense les grâces. Car, encore que ce grand ministre, choisi par la divine Providence pour présider aux conseils du plus sage de tous les rois, ait été le digne instrument des desseins les mieux concertés que l'Europe ait jamais vus; encore que la sagesse, après l'avoir gouverné dès son enfance, l'ait porté aux plus hauts honneurs et au comble des félicités humaines, sa fin nous a fait paraître que ce n'était pas pour ces avantages qu'il en écoutait les conseils. Ce que nous lui avons vu quitter sans peine n'était pas l'objet de son amour. Il a connu la sagesse que le monde ne connaît pas, cette sagesse qui "vient d'en haut, qui descend du Père des lumières », et qui fait marcher les hommes dans les sentiers de la justice. C'est elle dont la prévoyance s'étend aux siècles futurs, et enferme dans ses desseins l'éternité tout entière. Touché de ses immortels et invisibles attraits, il l'a recherchée avec ardeur, selon le précepte du Sage. «La sagesse vous élèvera, dit Salomon, et vous donnera de la gloire quand vous l'aurez embrassée. » Mais ce sera une gloire que le sens humain ne peut comprendre. Comme ce sage et puissant ministre aspirait à cette gloire, il l'a préférée à celle dont il se voyait environné sur la terre. C'est pourquoi sa modération l'a toujours mis audessus de sa fortune. Incapable d'être ébloui des grandeurs humaines, comme il y paraît sans ostentation, il y est vu sans envie; et nous remarquons dans sa conduite les trois caractères de la véritable sagesse, qu'élevé sans empressement aux premiers honneurs, il a vécu aussi modeste que grand; que dans ses importants emplois, soit qu'il nous paraisse comme chancelier, chargé de la principale administration de la justice, ou que nous le considérions dans les autres occupations d'un long ministère, supérieur à ses intérêts, il n'a regardé que le bien public; et qu'enfin dans une heureuse vieillesse, prêt à rendre avec sa grande âme le sacré dépôt de l'autorité si bien confié à ses soins, il a vu disparaître toute sa grandeur avec sa vie, sans qu'il lui en ait coûté un seul soupir : tant il avait mis en lieu haut et inaccessible à la mort son cœur et ses espérances! De sorte qu'il nous paraît selon la promesse du Sage, dans « une gloire immortelle », pour s'être soumis aux lois de la véritable sagesse, et pour avoir fait céder à la modestie l'éclat ambitieux des grandeurs humaines, l'intérêt particulier à l'amour du bien public, et la vie même au désir des biens éternels : c'est la gloire qu'a remportée très haut et puissant seigneur messire Michel Le Tellier, chevalier, chancelier de France. (Bossuet, 1961, p. 163-164)

Cette oraison se veut et elle doit être une apothéose, l'aboutissement sacré d'un crescendo vital dont le début se situe quatrevingt-trois ans plus tôt, en 1603, et dont la

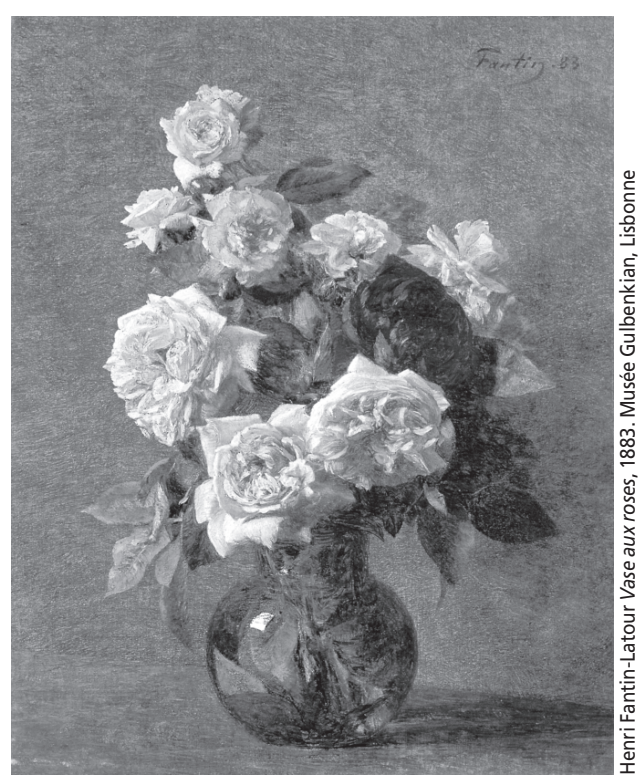

fin est marquée, selon Bossuet, par la «gloire immortelle » d'une mort chrétienne exemplaire. Dans la suite de son oraison, Bossuet souligne le rôle majeur joué par Le Tellier pendant la Fronde, mais il fait à peine allusion à ses trente-quatre longues années passées à la tête du ministère de la Guerre, alors que les historiens feront de Le Tellier le créateur même de l'armée monarchique. Par contre, il insiste longuement sur la signature par Louis XIV et Le Tellier de la révocation de l'édit de Nantes mettant fin aux privilèges accordés aux Protestants par Henri IV. C'est le chancelier Le Tellier qui avait préparé le texte de la révocation que Louis XIV a approuvé et amendé légèrement le 15 octobre 1685 . Le chancelier Le Tellier signa la document trois jours plus tard, soit le 18 octobre. Voltaire (1851, p. 418) écrira: «Le vieux chancelier Le Tellier, en signant l'édit, s'écria plein de joie: «Nunc dimittis servum tuum, Domine, quia viderunt oculi mei salutare tuum» ( Maintenant, seigneur, congédie ton serviteur car mes yeux ont vu que tu étais sauvé »). La révocation fut enregistrée par tous les parlements le 22 octobre 1685, soit huit jours seulement avant la mort du chancelier.

À ce moment-là, le Grand Siècle est à son pinacle et le clan Le Tellier, à son apogée ${ }^{4}$. La révocation de l'édit de Nantes met fin à l'exercice public du protestantisme en France en étendant à ce pays le principe du cujus regio, ejus religio que, depuis 1555, la paix d'Augsbourg a fait prévaloir en Allemagne. Cette mesure fait la quasiunanimité. Puissante, riche, triomphante et en paix, la France apparaît (faussement), en ce début de 1686, plus unie que jamais. Bourdaloue, Bossuet, Massillon, Fléchier, La Bruyère, La Fontaine se réjouissent publiquement. Même la marquise de Sévigné (1972, tome III, p. 239) s'est exclamée, le 28 octobre 1685 , en parlant du nouvel édit: «Rien n'est si beau que tout ce qu'il contient, et jamais aucun roi n'a fait et ne fera rien de plus mémorable.»

Bossuet, dans son oraison,

lui fait écho:

Poussons jusqu'au ciel nos acclamations, et disons à ce nouveau Constantin, à ce nouveau Théodose, à ce nouveau Marcien, à ce nouveau Charlemagne, ce que les six cent trente Pères dirent autrefois dans le concile de Chalcédoine: Vous avez affermi la foi, vous avez exterminé les hérétiques: c'est le digne ouvrage de votre règne, c'en est le propre caractère. Par vous, l'Hérésie n'est plus: Dieu seul a pu faire cette merveille. [...] Quand le sage chancelier reçut l'ordre de dresser ce pieux édit qui donne le dernier coup à l'Hérésie, il avait déjà ressenti l'atteinte de la maladie dont il est mort. [...] Seulement Dieu lui réservait l'accomplissement du grand ouvrage de la Religion; et il dit en scellant la révocation du fameux édit de Nantes, qu'après ce triomphe de la Foi et un si beau monument de la piété du Roi, il ne se souciait plus de finir ses jours.

(Bossuet, 1961, p. 183-184)

Comme Bossuet, Fléchier voit dans la participation du chancelier à la révocation de l'édit de Nantes un acte providentiel et l'aboutissement d'une longue vie chrétienne:

Quel zèle ne témoigna-t-il pas toujours pour l'Église, et par sa propre piété, et par les soins de ce fils [Charles-Maurice Le Tellier, archevêque-duc de Reims] qui en remplit les dignités avec éclat, et qui en soutient les droits avec fermeté?

Perdit-il une occasion, ou de maintenir 


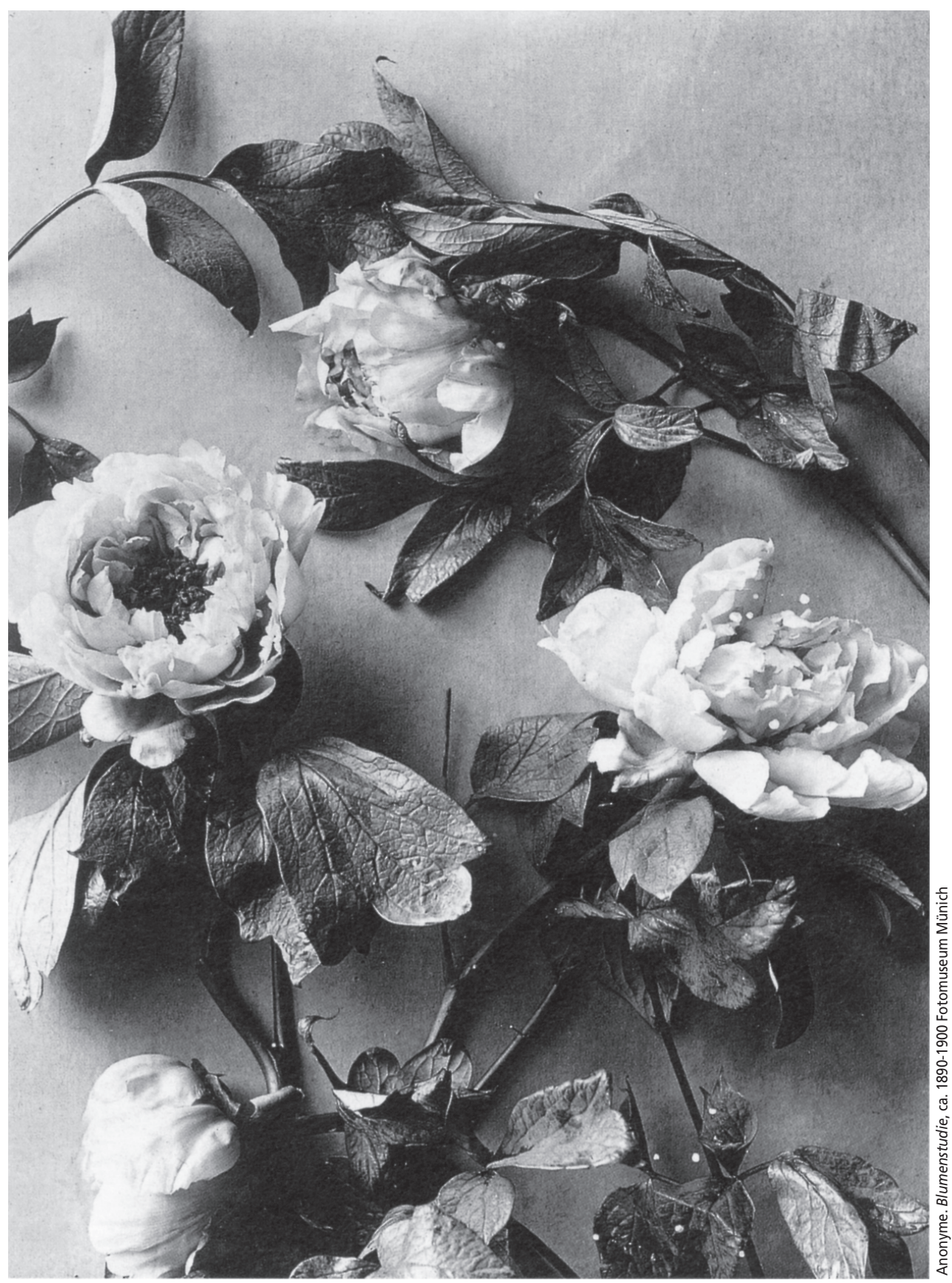

ses privilèges, ou de pacifier ses différends, ou d'appuyer sa discipline, et même d'étendre sa foi sur les débris heureux et inespérés de l'hérésie?

Quel spectacle s'ouvre ici à mes yeux, et où me conduit mon sujet! Je vois la droite du Très-Haut changer, ou du moins frapper les cœurs, rassembler les dispersions d'Israël, et couper cette haie fatale qui séparait depuis longtemps l'héritage de nos pères d'avec le nôtre. Je vois des enfants égarés revenir en foule au sein de leur mère ; la justice et la vérité détruire les œuvres de ténèbres et de mensonge ; une nouvelle église se former dans l'enceinte de ce royaume; et l'hérésie, née dans le concours de tant d'intérêts et d'intrigues, accrue par tant de factions et de cabales, fortifiée par tant de guerre et de révolte, tomber tout d'un coup, comme une autre Jéricho, au bruit des trompettes évangéliques, et de la puissance souveraine qui l'invite ou qui la menace.

Je vois la sagesse et la piété du prince, excitant les uns par ses pieuses libéralités, attirant les autres par les marques de sa bienveillance, relevant sa douceur par sa majesté, modérant la sévérité des édits par sa clémence, aimant ses sujets et haïssant leurs erreurs, ramenant les uns à la vérité par la persuasion, les autres à la charité par la crainte: toujours roi par autorité, et toujours père par tendresse.

Il ne restait qu'à donner le dernier coup à cette secte mourante; et quelle main était plus propre à ce ministère que celle de ce sage chancelier, qui, dans la vue de sa mort prochaine, ne tenant presque plus au monde, et portant déjà l'éternité dans son cœur, entre l'espérance et la miséricorde du Seigneur, et l'attente terrible de son jugement, méritait d'achever l'œuvre du prince, ou pour mieux dire l'œuvre de Dieu, en scellant la révocation de ce fameux édit, qui avait coûté tant de sang et tant de larmes à nos pères ? (Fléchier, 1774, p. 339-342)

Si Bossuet et Fléchier s'entendent pour célébrer le rôle de Le Tellier dans la révocation de l'édit de Nantes, ils choisissent de mettre en lumière des aspects différents du personnage. Bossuet souligne la sagesse, le souci du bien public, le sens politique et celui de la justice du chancelier défunt, tandis que Fléchier insiste sur ses contributions comme ministre de la Guerre et comme réformateur de l'enseignement du droit. Fléchier s'exprime de la façon suivante:

Ce fut dans ces révolutions [allusion à la mort de Richelieu et aux changements qui en ont découlé] que M. Le Tellier, contre les apparences et contre ses propres projets, fut rappelé de ses emplois, pour entrer dans la charge de secrétaire d'État, et dans le ministère de la guerre, en un temps où la discorde régnait dans toutes les parties de l'Europe, où le bruit de nos armes retentissait de tous côtés, et où nos ennemis et nos envieux s'animaient par nos pertes, et s'irritaient de nos victoires. Il fallait un homme laborieux pour se charger d'un long et pénible détail ; exact, pour entretenir l'ordre et la discipline de tant d'armées; fidèle, pour distribuer les finances avec des mains pures et innocentes; juste, pour représenter les services des soldats et des officiers, et faire élever les plus dignes aux places qu'une louable, mais malheureuse valeur rendait vacantes; sage, pour ménager dans des conjonctures difficiles ces esprits vains et remuants, qu'il est également dangereux d'abattre ou d'élever; éclairé, pour décider dans les conseils, et trouver des expédients et des ouvertures dans les affaires. (Fléchier, 1774, p. 319-321)

[...] Un roi [Louis XIII], dont la vie fut le règne de la religion et de la justice, pouvait-il, en mourant, faire un plus digne choix que celui de M. Le Tellier? Le Dieu des armées bénit aussitôt nos guerres en ses mains; la réputation de nos armes ne fit que croître; la perte d'un roi victorieux fut adoucie par le gain d'une bataille, et 
par une suite de victoires.

(Fléchier, 1774, p. 322)

[...] Rappelez-vous donc en votre mémoire ces guerres si renommées dont il fut le directeur et le ministre; cette paix fortunée dont il fut le solliciteur, et pendant le traité [de Nimègue] le dépositaire; ces conquêtes surprenantes, dont il avait été comme le prophète; ces négociations avantageuses, dont il fut et l'auteur et le conducteur par ses projets et par ses vues. Ajoutez à tous ces honneurs le témoignage d'un roi [Louis XIV] dont les paroles sont des oracles : «Que jamais homme sur toutes sortes d'affaires n'avait été de meilleur conseil. »(Fléchier, 1774, p. 330)

Après avoir fait l'éloge du ministre de la Guerre, Fléchier souligne la contribution de Le Tellier dans le rétablissement de l'enseignement du droit en sa qualité de chancelier. En avril 1679, Le Tellier avait rétabli officiellement, après quatre cent soixante ans, l'enseignement du droit civil à l'Université de Paris. Cet enseignement avait été interdit le 11 mai 1219 par une bulle du pape Honorius III. L'enseignement du droit rétabli, Le Tellier avait ordonné que les jeunes passent trois ans dans les écoles de droit avant de pouvoir exercer. De ce fait, Le Tellier peut, à juste titre, être considéré comme le père des facultés de droit actuelles de France et des pays de tradition française. Fléchier souligne l'importance de cette réforme :

Une téméraire jeunesse se jetait sans étude et sans connaissance dans les charges de la robe: on entrait dans le sanctuaire des lois en violant la première loi, qui veut qu'on soit instruit de sa profession. Pour obtenir les privilèges des jurisconsultes, il suffisait d'avoir de quoi les acheter; l'équité s'éteignait avec la science, et les fortunes des particuliers tombaient entre les mains de ces ignorants volontaires, à qui le pouvoir de les défendre était un titre pour les ruiner. Il rétablit les études, et fit revivre dans les écoles de droit ces exercices publics et solennels, et ces rigoureuses épreuves qui feront refleurir les lois et l'éloquence de nos pères. (Fléchier, 1774, p. 338)

\section{LA MORT}

Pour Bossuet, si la vie de Le Tellier est irréprochable, sa mort est sublime et, du point de vue chrétien ${ }^{5}$, elle constitue le couronnement même de cette vie. Nous touchons ici au thème central développé par Bossuet dans son oraison. La toute fin de celle-ci, qui constitue un pur chef-d'œuvre de littérature classique, exprime cela avec une force sublime:

Peuples, ne le pleurez plus; et vous qui, éblouis de l'éclat du monde, admirez le tranquille cours d'une si longue et si belle vie, portez plus haut vos pensées. Quoi donc! quatre-vingttrois ans passés au milieu des prospérités, quand il n'en faudrait retrancher ni l'enfance où l'homme ne se connaît pas, ni les maladies où l'on ne vit point, ni tout le temps dont on a toujours tant de sujet de se repentir, paraîtront-ils quelque chose à la vue de l'éternité où nous nous avançons à si grands pas? [...] Comptons donc comme très court, Chrétiens, ou plutôt comptons comme un pur néant tout ce qui finit, puisque enfin, quand on aurait multiplié les années au delà de tous les nombres connus, visiblement ce ne sera rien, quand nous serons arrivés au terme fatal. Mais peut-être que, prêt à mourir, on comptera pour quelque chose cette vie de réputation, ou cette imagination de revivre dans sa famille qu'on croira laisser solidement établie. [...] Dormez votre sommeil, riches de la terre, et demeurez dans votre poussière. Ah! si quelques générations, que dis-je? si quelques années après votre mort, vous reveniez, hommes oubliés, au milieu du monde, vous vous hâteriez de rentrer dans vos tombeaux, pour ne pas voir votre nom terni, votre mémoire abolie et votre prévoyance trompée dans vos amis, dans vos créatures, et plus encore dans vos héritiers et dans vos enfants. [...]

Surtout, mortels, désabusez-vous de la pensée dont vous vous flattez, qu'après une longue vie la mort vous sera plus douce et plus facile. Ce ne sont pas les années, c'est une longue préparation qui vous donnera de l'assurance. [...] C'est de saintes méditations, c'est de bonnes œuvres, c'est ces véritables richesses que vous enverrez devant vous au siècle futur, qui vous inspireront de la force; et c'est par ce moyen que vous affermirez votre courage. Le vertueux Michel Le Tellier vous en a donné l'exemple : la sagesse, la fidélité, la justice, la modestie, la prévoyance, la piété, toute la troupe sacrée des vertus qui veillaient, pour ainsi dire, autour de lui, en ont banni les frayeurs, et ont fait $\mathrm{du}$ jour de sa mort le plus beau, le plus triomphant, le plus heureux jour de sa vie. (Bossuet, 1961, p. 188-190).

Cette conclusion, qui se défend tout à fait dans le contexte de la théologie catholique, a heurté les auditeurs de Bossuet. Ces derniers étaient surtout venus assister au triomphe d'une certaine façon d'être honnête homme et à la célébration des valeurs familiales et patrimoniales. Moins que la mort d'un homme, c'est l'apothéose du clan Le Tellier alors au pinacle qu'on s'attendait à voir souligner. Michel Le Tellier, homme de nulle part, laissait derrière lui un sillage de pouvoir, d'alliances et de richesses. Ce grand héritage avait été bâti sans excès, sans vices et sans compromissions. Cette réussite même rassurait. Elle constituait un monument à la grandeur du Grand Siècle et à la solidité des valeurs qui l'inspiraient.

Mais voilà que Bossuet, cet ami de la famille Le Tellier, ce pilier du clan, rappelait la vanité de toute chose et l'incertitude de tout projet humain. C'est sur ce point, nous le verrons, que l'oraison prononcée par Fléchier se démarquera le plus de celle de Bossuet. Pour le moment, soulignons l'importance que revêtait, sous Louis XIV, une mort «chrétienne » préparée, entourée des sacrements de l'Église et, pour ainsi dire, «éveillée » comme celle du chancelier en la comparant à la mort de Louvois en 1691. Cette mort est décrite par Barbezieux, petitfils de Le Tellier et fils de Louvois, dans une lettre à René III de Froullay, comte de Tessé, datée du 21 juillet 1691 :

Il mourut lundi plus subitement que l'on ne peut se l'imaginer. Il s'était plaint un demi-quart d'heure auparavant d'avoir quelque chose dans l'estomac qui l'étouffait. L'on le saigna du côté gauche, et se sentant soulagé par cette saignée, il demanda qu'on en fit autant de l'autre bras. Son médecin lui refusa, par l'extrême faiblesse où il était. Il demanda où j'étais, et qu'on m'allât quérir; j'étais malade dans mon lit; l'on me fit avertir. M. Fagon, pour qui il avait beaucoup de considération, sur les entrefaites entra dans sa chambre. Il commença à lui conter ce qui lui faisait mal; mais, un moment après, il dit qu'il étouffait. Il me demanda encore avec empressement, et il dit qu'il se mourait. Après ces dernières paroles, la tête lui tomba sur les épaules, ce qui fut le dernier moment de sa vie. J'arrivai comme la tête lui tombait, et voyant tout le monde désolé, et ne pouvant croire ce que le triste visage d'un chacun m'apprenait, je me jetai à lui ; mais il était insensible à mes caresses, et c'en était déjà fait. L'on l'a ouvert le lendemain, et, quoiqu'il n'y ait point d'indice assez positif pour assurer qu'il ait été empoisonné, il n'y a cependant presque pas lieu d'en douter. Voilà comment j'ai perdu tout ce qui m'était le plus cher au monde. (Rousset, 18611864, tome IV, p. 498) 
Quel contraste entre ce récit et celui de la mort du chancelier Le Tellier! Le père est mort en patriarche, entouré de sa famille, en paix avec l'Église et avec la société, après avoir reçu les sacrements et confessé ses péchés; le fils meurt subitement, sans préparation, sans secours religieux, sans famille, mais en ayant cependant le temps de se rendre compte qu'il meurt. Pour l'homme du XVII ${ }^{\mathrm{e}}$ siècle, autant la mort du premier est sublime, autant celle du second est horrible. Charles Perrault (l'auteur des Contes de ma mère l'Oye) exprimera cela dans une épitaphe:

Figure du monde qui passe

Et qui passe dans un moment

Pompe, richesse, honneur, funeste amusement,

Dont un mortel s'enivre et ne jamais se lasse,

De quoi sert votre éclat à l'heure de la mort?

Il ne peut ni changer ni retarder le sort.

Louvois plus haut que lui ne voyait que son maître ;

Dans le sein des grandeurs, des biens et des plaisirs,

Un trait fatal et prompt borne enfin ses désirs,

Et ne lui laisse pas le temps de se connaître.

Hélas! aux grands emplois à quoi sert de courir?

Pour veiller sur soi-même heureux qui s'en délivre !

Qui n'a pas le temps de bien vivre

Trouve malaisément le temps de bien mourir.

\section{LA POSTÉRITÉ}

L'idée que l'individu puisse se prolonger à travers sa descendance se trouve à la base même du système aristocratique qui sacralise les concepts de famille et de postérité (l'anoblissement de l'individu s'étendant à toute sa postérité), mais elle se trouve aussi au cœur du fonctionnement de l'État monarchique dans la mesure où la noblesse d'épée qui constitue l'un des fondements essentiels de cet État tire une grande partie de son courage, de son abnégation et de son audace au combat de la conviction qu'elle a que la mort de l'individu noble n'est au fond que peu de chose puisque la famille et la lignée nobles se perpétuent. Cela dit, le dogme chrétien entre parfois en contradiction avec cette vision des choses, comme nous le font voir Bossuet et Fléchier qui posent directement les grandes questions du sens de la vie, de la mort et de l'idée de postérité en se penchant sur le cas du fondateur même d'un des plus illustres clans de l'histoire de France.

Au moment où Bossuet et Fléchier prononcent leurs oraisons, le clan Le Tellier monopolise presque le pouvoir ${ }^{6}$. En exerçant les premières responsabilités dans le domaine de la justice, le clan Le Tellier contrôle, en bonne partie, la noblesse de robe ; par le secrétariat d'État de la guerre, il contrôle la noblesse d'épée. Enfin, par le frère de Louvois, Charles-Maurice Le Tellier, archevêque-duc de Reims, et par son allié, Bossuet, le clan exerce une influence considérable sur l'Église de France.

Tant de puissance léguée par le chancelier Le Tellier ne peut qu'amener l'assemblée réunie pour entendre le panégyrique de Bossuet à déplorer qu'au milieu de l'éloge de tant de vertus, l'orateur sacré n'ait pu trouver une place pour la célébration d'une réussite terrestre presque sans égal dans ce siècle. La rigueur chrétienne du sermon de Bossuet et sa sévérité ont désarmé l'assemblée. Une remarque de La Bruyère montre que cette réaction n'avait rien d'exceptionnel à cette époque. En effet, La Bruyère écrit: "Ce qu'on appelle une oraison funèbre n'est aujourd'hui bien reçue du plus grand nombre des auditeurs, qu'à mesure qu'elle s'éloigne davantage du discours chrétien, ou, si vous l'aimez mieux ainsi, qu'elle approche de plus près d'un éloge profane» (La Bruyère, 1951, p. 443).

Là où, au scandale de plusieurs, Bossuet avait choisi de présenter comme la vanité suprême le désir de vouloir survivre dans sa descendance, Fléchier choisit de célébrer la vertu du défunt chancelier en le présentant comme un patriarche et en rendant hommage à sa postérité. D'entrée de jeu, l'oraison prononcée par Fléchier annonce ses couleurs :

Sa vertu s'est soutenue jusqu'à sa vieillesse; elle l'a fait monter aux lieux élevés de la terre : sa postérité a recueilli son héritage, afin que les enfants d'Israël connaissent qu'il est bon d'obéir au Dieu saint.

(Ecclésiaste, 46)

[...] Dans l'éloge que je fais aujourd'hui de très haut et puissant seigneur Messire Michel Le Tellier, ministre d'État, chevalier, chancelier de France, j'envisage, non pas sa fortune, mais sa vertu; les services qu'il a rendus, non pas les places qu'il a remplies; les dons qu'il a reçus du ciel, non pas les honneurs qu'on lui a rendus sur la terre; en un mot, les exemples que votre raison vous doit faire suivre, et non pas les grandeurs que votre orgueil pourrait vous faire désirer [...]
Il est vrai que le ciel a rempli ses désirs, et qu'il a eu, pour ainsi dire, la destinée des patriarches ; cette plénitude de jours qui consomme la prudence de l'homme juste; cette suite de bons succès que le temps et la fortune qui change tout, n'ont osé troubler; ces richesses innocentes qui ont entretenu son honnête et frugale opulence; cet esprit qui, malgré le poids des années et des affaires, a conservé sa force et sa vigueur dans les ruines mêmes du corps; cette gloire qu'il a maintenue et qu'il a vu renaître en ses enfants de génération en génération; cette mort dans la paix et dans l'espérance du seigneur, qu'il a regardée comme la fin de son travail et le terme de son pèlerinage. (Fléchier, 1774, p. 305-308)

En soulignant que le ciel a rempli les désirs de Michel Le Tellier en lui réservant la destinée des patriarches, Fléchier vise juste: on attribue deux devises à Le Tellier qui, toutes deux, parlent de postérité. Ce sont: "Virtus avorum durat cum sanguine» ( «la force morale des ancêtres se perpétue avec le sang») et «Invito funere vivent» ( «ils vivront grâce à des funérailles qui ne sont pas voulues», cette devise ayant un pélican pour cimier).

Dans une ultime tentative de réconciliation de la morale chrétienne avec l'idée de postérité, Fléchier louange l'homme privé et ses qualités de "père de famille», thème que Bossuet avait complètement ignoré :

Mais quelle était cette douceur, quand elle se renfermait dans l'enceinte de sa famille, et dans les bornes d'une vie privée! Quel sage et noble repos! Quelle tendresse pour ses enfants! Quelle union avec cette épouse fidèle, qui, selon le langage du Saint-Esprit, est la récompense de l'homme de bien! Quelle sensibilité et quelle constance pour ses amis! Qu'il eût aimé à jouir en repos du fruit de ses travaux dans une heureuse vieillesse! Il laissait à l'État un fils [Louvois] dont il avait formé l'esprit et le cœur; ils [Le Tellier et son fils, Louvois] remplissaient les mêmes emplois avec les mêmes vertus ; et ils auraient été l'un et l'autre inimitables, si le père n'eût eu le fils pour successeur, et si le fils n'eût eu le père pour exemple.

Ayant suppléé aux omissions et aux manques d'égards à la postérité du chancelier de l'oraison funèbre de Bossuet, Fléchier termine sa propre oraison en réconciliant les uns et les autres. Il évoque l'oraison de Bossuet et s'adresse à l'assistance : 
Sacré ministre de Jésus-Christ

[Fléchier s'adresse ici directement à Bossuet qui officie], qui, dans la chaire évangélique, avec une éloquence vive et chrétienne, avez avant moi consacré la mémoire immortelle de ce grand homme, achevez d'offrir pour lui cette hostie innocente et pure qui lave les péchés et les fragilités du monde. Peuple qui ressentez encore les effets de son exacte équité, reprenez le cantique qu'il avait commencé des miséricordes éternelles. Et vous, vaillants et malheureux guerriers, qui, dans cet hôtel royal [des Invalides], traînant les restes de vos corps aux pieds de ces autels, attendant avec patience une mort que vous avez si souvent bravée, sacrifiez au Dieu de la paix les lauriers que vous avez cueillis dans les armées, et faites des malheurs de votre ambition et de votre gloire, les fruits de votre pénitence: redoublez, pour son repos éternel, ces vœux ardents que vous avez si souvent faits pour une vie si utile et si précieuse. (Fléchier, 1774, p. 345-346)

\section{RÉVOCATION ET RÉVOLUTION}

Ce que fait surtout ressortir la comparaison des deux oraisons funèbres, c'est que, malgré l'unanimité des éloges, des fissures apparaissaient dans la morale du Grand Siècle. La vision proprement théologique adoptée par Bossuet face à la mort qui transcende la vie et la postérité a heurté la société de son temps, ce qui révélait une fissure idéologique latente que Fléchier a tenté, tant bien que mal, de calfeutrer en se proposant de réconcilier les trois éléments du triangle vie-mort-postérité.

Si Bossuet conteste la valeur de la postérité dans la perspective chrétienne, le siècle qui suivra remettra en question la valeur de cette même idée de postérité du point de vue social. Cela débouchera directement sur la Révolution française et sur le rejet de l'idéal aristocratique d'une société basée sur les familles et sur la transmission de père en fils aîné des titres et des privilèges.

Assez curieusement, cette remise en question sera beaucoup plus profonde que celle de l'idéal unificateur de la révocation de l'édit de Nantes. Il ne faut jamais oublier que la couleur jacobine que prendra la Révolution découle du même esprit qui a donné naissance à la révocation. De ce point de vue, il n'est pas excessif de dire, à la lumière de ce qui précède, que la dimension philosophique de la Révolution française peut avoir été plus révolutionnaire que sa dimension proprement sociale puisqu'en somme, c'est bel et bien le lien philoso- phique unissant les concepts de vie, de mort et de postérité qui a été revu et corrigé lors de la Révolution.

Le débat engendré par les oraisons funèbres de Le Tellier annonçait tout cela. De ce point de vue, l'oraison funèbre prononcée par Bossuet était profondément révolutionnaire bien que, dans l'esprit de son auteur, elle se voulait avant tout conforme à la morale chrétienne. Quant à Fléchier, en voulant plaire à son auditoire, il n'a au fond que tenté de sauver un système de valeurs que la Révolution fera s'écrouler ... temporairement.

\section{Bibliographie}

BOSSUET, Jacques-Bénigne (1961). «Oraison funèbre de Messire Michel Le Tellier, Chevalier, Chancelier de France», Euvres: oraisons funèbres, panégyriques, Textes établis et annotés par l'Abbé Bernard Velat et Yvonne Champailler, Paris, Gallimard, Bibliothèque de la Pléiade, p. 163-190.

FLÉCHIER, Esprit (1774). «Oraison funèbre de Très-Haut et Puissant Seigneur Messire Michel Le Tellier, Chevalier, Chancelier de France", Recueil des oraisons funèbres prononcées par messire Esprit Fléchier, évêque de Nîmes, Paris, Saillant et Nyon, p. 305-346.

HERSAN (1686). Oraison funèbre de Très Haut et Très Puissant seigneur Michel Le Tellier, traduit du latin par Noël Bosquillon, Paris, édit. 1802.

LA BRUYÈRE, Jean de (1951). Euvres complètes, Paris, Gallimard, Bibliothèque de la Pléiade, $739 \mathrm{p}$.

MABOUL, Jacques (1686). Oraison funèbre de Michel Le Tellier, Paris.

ROUSSET, Camille (1861-1864). Histoire de Louvois et de son administration politique et militaire, Paris, Didier et Cie, 4 volumes.

SÉVIGNÉ, Marie de Rabutin-Chantal, marquise de (1972). Correspondance, Paris, Gallimard, Bibliothèque de la Pléiade, 3 tomes.

TELLIER, Luc-Normand (1987). Face aux Colbert: les Le Tellier, Vauban, Turgot ... et l'avènement $d u$ libéralisme, Québec, Presses de l'Université du Québec, 806 p.

VOLTAIRE (1851). Siècle de Louis XIV, Paris, Librairie de Firmin Didot frères.

\section{Notes}

1. L'histoire de ce clan dont ont fait partie Bossuet, Vauban, Turgot, Condorcet et Tocqueville, entre autres, a été retracée par Tellier (1987).

2. Le duc de Richelieu, Premier ministre sous Louis XVIII, descendait directement de Le Tellier, de son fils, Louvois, et du fils de Louvois, Barbezieux, qui ont tous été ministres sous Louis XIV. Parmi les descendants de Le Tellier et de Louvois qui ont été ministres, mentionnons aussi Louis-CharlesCésar Le Tellier, maréchal-duc d'Estrées, ministre d'État sous Louis XV.
3. Le 25 janvier 1686, Bossuet a prononcé l'oraison funèbre du chancelier Le Tellier dans l'église Saint-Gervais à Paris. Le 8 février 1686, Hersan, "professeur royal de l'éloquence», en prononça une en latin dans l'église de la Sorbonne, "au service de l'université », dont le fils du chancelier, Charles-Maurice Le Tellier, archevêque-duc de Reims, deviendra, en 1695, le proviseur. Un autre éloge en latin a été composé par les religieux de l'abbaye de Saint-Germaindes-Prés. Le 2 mars 1686, ce fut au tour de Jacques Maboul, évêque d'Alet, de faire l'oraison funèbre du chancelier, en l'église des Grands Augustins. Enfin, le 22 mars 1696, dans l'église des Invalides, construites par les soins de Louvois, devant un auditoire de militaires et en présence de Bossuet qui officiera, Esprit Fléchier, évêque de Nîmes, prononça une dernière oraison funèbre qui réjouit tout le monde et fit l'unanimité.

4. Depuis le traité de Nimègue en juillet 1678, le royaume de France comprend la Franche-Comté et une partie des Pays-Bas espagnols. En septembre 1681, sans effusion de sang, Louvois a réuni Strasbourg à la France. La paix règne sur la France et la France, sur l'Europe

5. Du moins du point de vue de la théologie catholique suivant laquelle le salut du pécheur se décide à l'heure de sa mort, l'assistance salvatrice de l'Église devant être sollicitée jusqu'au tout dernier moment. Évidemment, la vision calviniste marquée par la prédestination est tout autre.

6. Louvois occupe les fonctions de secrétaire d'État de la guerre qu'il a héritées de son père, de surintendant des postes, depuis le 24 décembre 1668, et de surintendant des bâtiments, des arts et des manufactures, depuis le 8 septembre 1683. Son fils, Barbezieux, détient la survivance du secrétariat d'État de la guerre depuis le 13 novembre 1685 , soit quelques jours après la mort du chancelier Le Tellier, en remplacement de son frère, Courtanvaux, qui l'avait obtenue le 7 décembre 1681. Leur cousin, Claude Le Peletier, a succédé à Jean-Baptiste Colbert au poste de contrôleur général des finances. Michel Le Tellier a occupé les fonctions de chancelier de France et de garde des sceaux du 27 octobre 1677 à sa mort, le 30 octobre 1685. Louis Boucherat lui succède qui ne s'oppose en rien aux Le Tellier. Face à cette famille toute-puissante se maintient péniblement le clan Colbert, qui, bien qu'issu du clan Le Tellier, a longtemps cherché à le supplanter. Jean-Baptiste Colbert, l'illustre contrôleur général des finances, est mort le 6 septembre 1683. La même année, son neveu, Nicolas Desmaretz, a été disgracié. Restent le fils de Colbert, Seignelay, au secrétariat d'État de la marine et son frère, Croissy, au secrétariat d'État des affaires étrangères. Le pouvoir réel de ces deux survivants du clan Colbert est cependant limité, car l'omniprésence de Louvois se fait peutêtre encore plus sentir, en ce temps de paix, dans le domaine de la politique étrangère et de la diplomatie que n'importe où ailleurs. 